\title{
Block Five Diagonal Matrices and the Fast Numerical Solution of the Biharmonic Equation ${ }^{1}$
}

\author{
By Louis Bauer and Edward L. Reiss
}

\begin{abstract}
A factoring and block elimination method for the fast numerical solution of block five diagonal linear algebraic equations is described. Applications of the method are given for the numerical solution of several boundary-value problems involving the biharmonic operator. In particular, 22 eigenvalues and eigenfunctions of the clamped square plate are computed and sketched.
\end{abstract}

1. Introduction. The system of linear algebraic equations

$$
M \mathbf{w}=\mathbf{r}
$$

is of block five diagonal form if the matrix $M$ is given by

$$
\begin{aligned}
M & =\left[A_{i}, B_{i}, C_{i}, D_{i}, E_{i}\right] \\
& \equiv\left(\begin{array}{cccccccc}
C_{1} & D_{1} & E_{1} & 0 & . & . & . & 0 \\
B_{2} & C_{2} & D_{2} & E_{2} & 0 & . & . & 0 \\
A_{3} & B_{3} & C_{3} & D_{3} & E_{3} & 0 & . & 0 \\
0 & A_{4} & B_{4} & C_{4} & D_{4} & E_{4} & 0 & 0 \\
& 0 & . & \cdot & & & & \\
\vdots & \vdots & & & \vdots & \vdots & \vdots & \vdots \\
0 & \cdot & . & 0 & A_{q-1} & B_{a-1} & C_{q-1} & D_{q-1} \\
0 & . & . & . & 0 & A_{q} & B_{q} & C_{a}
\end{array}\right) .
\end{aligned}
$$

Each of the matrices $A_{i}, B_{i}, C_{i}, D_{i}$ and $E_{i}$ has $p_{i}$ rows and each of the matrices $E_{i-2}, D_{i-1}, C_{i}, B_{i+1}$ and $A_{i+2}$ has $p_{i}$ columns. Thus, the main diagonal matrices $C_{i}$ are square and $M$ is square of dimension $\nu \equiv \sum_{i=1}^{q} p_{i}$.

Linear algebraic equations of the form (1.1) are obtained, for example, from difference approximations of boundary-value problems for fourth-order elliptic partial differential equations. A specific example is

Received June 4, 1971.

AMS 1970 subject classifications. Primary 65N20, 65N25, 65F05, 65F10, 65F15; Secondary 73K 10.

Key words and phrases. Block five diagonal matrix, factoring method, block elimination procedure, biharmonic equation, biharmonic boundary-value problem, clamped elastic plate, eigenvalues, eigenfunctions, clamped cylindrical panel, perturbation expansion.

${ }^{1}$ The research reported in this paper was supported by the U. S. Atomic Energy Commission under Contract No. AT(11-1)-3077 and the National Science Foundation under Grant No. GP-27223. The authors benefited from helpful discussions with Professor $O$. Widlund. 


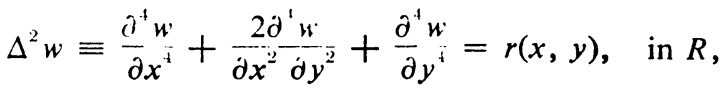

$$
\begin{aligned}
& \boldsymbol{w}=\mathbf{n} \cdot \nabla \boldsymbol{w}=\mathbf{0},
\end{aligned}
$$

Here, $R$ is a region of the $x, y$ plane, $B$ is the boundary of $R, \mathrm{n}$ is the unit vector normal to $B$ and $r(x, y)$ is a prescribed function on $R$.

When $R$ is a rectangle and the standard 13-point difference approximation of the biharmonic operator is employed, then (1.2) is equivalent to the algebraic system (1.1), where $p_{1}=p_{2}=\cdots=p_{a}=p$, and

$$
\begin{array}{ll}
C_{1}=C_{a}=C+I, & \\
C_{i}=C, & j=2, \cdots, q-1, \\
B_{i}=D_{i-1}=B, & j=2, \cdots, q, \\
A_{i}=E_{i-2}=I, & j=3, \cdots, q .
\end{array}
$$

Here, $I$ is the unit $p \times p$ matrix. The $p \times p$ matrices $B$ and $C$ are of scalar three and scalar five diagonal form, respectively. They are defined by

$$
\begin{aligned}
& B \equiv[0,2,-8,2,0], \\
& C \equiv\left[1,-8, c_{k},-8,1\right], \quad c_{k}= \begin{cases}21, & \text { if } k=1, p, \\
20, & \text { if } k=2, \cdots, p-1 .\end{cases}
\end{aligned}
$$

In (1.3b), we have applied the notation of (1.1b) to scalar matrices. Other difference approximations of (1.2), which may be obtained, for example, by the finite element method, may also yield algebraic equations of block five diagonal form. Specifically, the algebraic equations obtained from the 25-point difference approximation of (1.2) are of the form (1.1).

There are nonlinear problems and more complicated linear problems which involve coupled systems of equations like (1.2), cf. Section 6. Approximate solutions of these problems can be obtained by iterative procedures. Then, at each step, (1.2) must be solved one or more times, $r$ being determined from the previous iterates. Since many iterations may be required for convergence, methods for the fast numerical solution of (1.2), and therefore (1.1), are desirable. In this paper, we present such a method. It is a direct method which is an extension to block five diagonal matrices of the factoring method previously suggested for block three diagonal matrices [1].

The unit inversion time for the solution of (1.1) by this method is significantly less than that for iterative methods such as the alternating direction method [2]. This speed is achieved at the expense of large fast-access memory storage requirements. In Section 3, we present a modification of the method by applying a block elimination procedure which reduces the storage requirement. This procedure is related to the one proposed for harmonic boundary-value problems [3].

Applications of the factoring and block elimination methods are given in Sections 4-6. In Section 4, the numerical results are compared with exact solutions and with numerical solutions obtained by other methods. In Section 5, we apply the present methods and Richardson's mesh extrapolation procedure to obtain accurate estimates of 22 eigenvalues (counting multiplicities) and eigenfunctions for the flexural vibrations 
of clamped, square, elastic plates. The first 6 eigenvalues are in excellent agreement with the results of the Ritz method [4], [5]. However, the present numerical results show that the principal eigenfunction has nodal curves near the corners of the plate. This seems not to have been observed previously. Finally, in Section 6, we obtain numerical approximations for the lowest eigenvalue of the shallow, clamped cylindrical panel. They are in excellent agreement with the values obtained by the perturbation method described in the Appendix. Another application of the method is given in [6].

2. The Factoring Method. We factor the matrix $M,(1.1 \mathrm{~b})$, into the product of an upper block triangular matrix $U$ and a lower block triangular matrix $L$. That is,

$$
M=L U
$$

where, using the notation in (1.1b), $L$ and $U$ are given by

$$
\begin{aligned}
L & =\left[\alpha_{i}, \beta_{i}, \gamma_{i}, 0,0\right], \\
U & =\left[0,0, I_{i}, \delta_{i}, \eta_{i}\right] .
\end{aligned}
$$

The unit matrices $I_{i}$ are $p_{i} \times p_{i}$. The matrices $\alpha_{i}, \beta_{i}, \gamma_{i}, \delta_{i}$ and $\eta_{i}$ are determined by substituting (2.2) into (2.1) and then comparing both sides of the equation. We find that

$$
\begin{aligned}
\alpha_{i} & =A_{i}, & i & =3, \cdots, q, \\
\beta_{i} & =B_{i}-A_{i} \delta_{i-2}, & i & =2, \cdots, q, \\
\gamma_{i} & =C_{i}-\beta_{i} \delta_{i-1}-A_{i} \eta_{i-2}, & i & =1,2, \cdots, q, \\
\delta_{i} & =\gamma_{i}^{-1}\left[D_{i}-\beta_{i} \eta_{i-1}\right], & i & =1,2, \cdots, q-1, \\
\eta_{i} & =\gamma_{i}^{-1} E_{i}, & i & =1,2, \cdots, q-2 .
\end{aligned}
$$

where we define $\delta_{i}=\eta_{i} \equiv 0$ for $i \leqq 0$. Thus, the factorization is possible if $\gamma_{1}, \gamma_{2}, \cdots, \gamma_{a-1}$ are nonsingular matrices.

To solve (1.1), we first substitute (2.1) into (1.1) and define the vector $\nabla$ by

$$
U \mathbf{w}=\mathbf{v} \text {. }
$$

Then, $(1.1)$ is reduced to

$$
L \mathbf{v}=\mathbf{r} .
$$

Since the systems (2.4) and (2.5) are of upper and lower block triangular form, respectively, they can be solved directly. We partition $\mathbf{r}, \mathbf{v}$, and $\mathbf{w}$ into subvectors to conform with the partitioning of $L$ and $U$. Then the solution of (2.5), the forward sweep, is

$$
\mathbf{v}_{i}=\gamma_{i}^{-1}\left(\mathbf{r}_{i}-\beta_{i} \mathbf{v}_{i-1}-A_{i} \mathbf{v}_{i-2}\right), \quad i=1,2, \cdots, q,
$$

where $\mathbf{v}_{i} \equiv 0$ for $i \leqq 0$. The solution of (2.4), the backward sweep, is

$$
\mathbf{w}_{i}=\mathbf{v}_{i}-\delta_{i} \mathbf{w}_{i+1}-\eta_{i} \mathbf{w}_{i+2}, \quad i=q, q-1, \cdots, 1,
$$

where $\mathrm{w}_{i} \equiv 0$ for $i>q$.

Thus, only the intermediate matrices $\gamma_{i}^{-1}, \beta_{i}, A_{i}, \delta_{i}$, and $\eta_{i}$ are required to determine $w$. In the repeated solution of (1.1) for different vectors $r$, these matrices need 
only be computed once. To achieve greater speed in the computations, they are stored in the fast-access (core) memory of the computer. The solution formulas (2.6) and (2.7) involve only matrix-vector multiplications for which efficient machine language subroutines have been developed.

We shall use a unit inversion time $\tau$ to discuss the efficiency of the method. It is defined as the time required to evaluate $w$ from (2.6) and (2.7) for a fixed vector $r$. Thus, $\tau$ does not include the computing time necessary to generate and factor $M$, since this occurs only initially.

The large storage requirement is clearly a disadvantage of the method, since it restricts the size of the matrix $M$ which can be considered. The intermediate matrices could be stored in a slow-access memory device such as a tape or a disc. Then, significantly larger matrices could be accommodated, but the large increase in $\tau$ would be inconsistent with the purpose of the method.

To give an indication of typical storage requirements, we consider the system (1.1), (1.3). Since $A_{i}=E_{i}=I$, and $\eta_{i}=\gamma_{i}^{-1}$, we need store only the matrices $\beta_{i}$, $\gamma_{i}^{-1}$, and $\delta_{i}$. The total storage requirements for these matrices, when $p=q=25$ are 15000,15625 , and 15000 words, respectively. ${ }^{2}$

The speed and accuracy of the method were evaluated by obtaining numerical solutions of several boundary-value problems involving the biharmonic operator on the unit square. In all problems, the boundary conditions (1.2b) were satisfied and the 13-point biharmonic approximation was employed. For other boundary conditions, such as $w=w_{n n}=0$, where $w_{n n}$ indicates the second normal derivative, it may be possible to factor the biharmonic operator into the product of two harmonic operators. Then, the solution could be obtained by successively inverting two-block three-diagonal systems.

3. The Block Elimination Procedure. We shall describe the elimination procedure for (1.1) with $p_{1}=p_{2}=\cdots=p_{q}=p$, and

$$
\begin{array}{ll}
A_{i}=E_{i-2}=I, & j=3,4, \cdots, q, \\
B_{i}=D_{i}=B \text { and } C_{i}=C, & j=3,4, \cdots, q-2, \\
D_{2}=B_{q-1}=B . &
\end{array}
$$

Here, $B$ and $C$ are not necessarily the matrices defined in (1.3b). We denote this special system by

$$
M_{0} \mathbf{w}=\mathbf{r} .
$$

The matrices defined in (1.3) are included in (3.1). We shall first eliminate the even indexed subvectors $w_{2}, w_{4}, \cdots$ from (3.2). Since we may wish to repeat the procedure, it is convenient to simplify the elimination formulas by assuming that $q$ is odd and given by

$$
q=2^{k}+1
$$

We consider the equations in (3.2) corresponding to the five blocks $j-2, \cdots$, $j+2$ for any odd integer $j$ in the range, $5 \leqq j \leqq q-4$ :

${ }^{2} p=25$ corresponds to a mesh width of $\delta=1 / 26$ in the difference approximation of (1.2) and $q=p$ means a square region. 
$\mathbf{w}_{i-4}+B w_{i-3}+C w_{i-2}+B w_{i-1}+w_{i}$

$=\mathbf{r}_{i-2}$,

$w_{i-3}+B w_{i-2}+C w_{i-1}+B w_{i}+w_{i+1}$

$=\mathbf{r}_{i-1}$,

$$
\begin{aligned}
\mathbf{w}_{i-2}+B \mathbf{w}_{i-1}+C \mathrm{w}_{i}+B \mathrm{w}_{i+1}+\mathbf{w}_{i+2} & =\mathbf{r}_{i}, \\
\mathbf{w}_{i-1}+B \mathbf{w}_{i}+C \mathbf{w}_{i+1}+B \mathbf{w}_{i+2}+\mathbf{w}_{i+3} & =\mathbf{r}_{i+1}, \\
\mathbf{w}_{i}+B \mathbf{w}_{i+1}+C \mathbf{w}_{i+2}+B \mathbf{w}_{i+3}+\mathbf{w}_{i+4} & =\mathbf{r}_{i+2} .
\end{aligned}
$$

We multiply the second and fourth equations by $-B$ and the third equation by $K \equiv B C B^{-1}$ and add the resulting five equations. This yields a reduced system involving only the odd indexed subvectors. Equations equivalent to (3.4) are considered for $j=1,3, q-2$, and $q$ separately. Special elimination formulas are then required. The resulting system of equations is

$$
M_{0}^{1} \mathbf{w}^{1}=\mathbf{r}^{1},
$$

where $M_{0}^{1}, \mathrm{w}^{1}$, and $\mathrm{r}^{1}$ are defined below. The matrix $M_{0}^{1}$ is of the same form as $M_{0}$. The elimination can therefore be repeated. After the $(j+1)$ st elimination, there are $q_{i+1}$ block rows in the resulting matrix $M_{0}^{i+1}$, where

$$
q_{i+1}=\left(q+2^{j+1}-1\right) / 2^{j+1}
$$

and

$$
M_{\mathrm{n}}^{i+1} \mathbf{w}^{i+1}=\mathbf{r}^{i+1} .
$$

Here,

$$
\mathbf{w}_{i}^{j+1}=\mathbf{w}_{2 i-1}^{i}, \quad i=1,2, \cdots, \dot{q}_{i+1} .
$$

The submatrices of $M_{0}^{i+1}$ are recursively defined by, ${ }^{8}$

$$
\begin{aligned}
& C^{i+1}=2\left[I-\left(B^{i}\right)^{2}\right]+K^{i} C^{i}, \\
& B^{i+1}=C^{i}-\left(B^{i}\right)^{2}+K^{i},
\end{aligned}
$$

for the general matrices, and

$$
\begin{aligned}
& C_{1}^{j+1}=B^{i}\left(C_{2}^{i}-I\right)\left(D_{1}^{i}\right)^{-1} C_{1}^{i}-B^{i} B_{2}^{i}+I, \\
& C_{2}^{i+1}=B^{i}\left(C_{2}^{i}-C^{i}+I\right)\left(D_{1}^{i}\right)^{-1}-2\left(B^{i}\right)^{2}+K^{i} C^{i}+I, \\
& D_{1}^{i+1}=B^{i}\left(C_{2}^{i}-I\right)\left(D_{1}^{i}\right)^{-1}-\left(B^{i}\right)^{2}+C^{i}, \\
& B_{2}^{j+1}=B^{i}\left(C_{2}^{i}-C^{i}+I\right)\left(D_{1}^{i}\right)^{-1} C_{1}^{i}-B^{i} B_{2}^{i}+K^{i},
\end{aligned}
$$

for the special matrices. The formulas for the special matrices $C_{a}^{i+1}, C_{a-1}^{i+1}, B_{a}^{i+1}$, and $D_{a-1}^{i+1}$ can be obtained from (3.9b) in an obvious manner.

The reduced subvectors $r_{i}^{i+1}$ are given by

$$
\begin{aligned}
& \mathbf{r}_{1}^{i+1}=B^{i}\left(C_{2}^{i}-I\right)\left(D_{1}^{i}\right)^{-1} \mathbf{r}_{1}^{j}-B^{i} \mathbf{r}_{2}^{i}+\mathbf{r}_{3}^{j} \\
& \mathbf{r}_{2}^{j+1}=B^{i}\left(C_{2}^{i}-C^{i}+I\left(D_{1}^{j}\right)^{-1} \mathbf{r}_{1}^{i}-B^{i} \mathbf{r}_{2}^{j}+K^{i} \mathbf{r}_{3}^{j}-B^{j} \mathbf{r}_{4}^{j}+\mathbf{r}_{5}^{j},\right. \\
& \mathbf{r}_{i}^{i+1}=\mathbf{r}_{2 i-3}^{j}-B^{i} \mathbf{r}_{2 i-2}^{j}+K^{i} \mathbf{r}_{2 i-1}^{j}-B^{i} \mathbf{r}_{2 i}^{i}-\mathbf{r}_{2 i+1}^{j}, \quad i=3,4, \cdots, \bar{q}_{i+1}-2 .
\end{aligned}
$$

The superscripts in (3.9)-(3.11) are indices that indicate the number of eliminations. 
The equations for the last two subvectors of $r^{i+1}$ are obtained in an obvious way from the first two equations in (3.10). and

The eliminated vectors $\mathrm{w}_{2}^{i}, \mathrm{w}_{4}^{i}, \cdots, \mathrm{w}_{a_{i-1}}^{i}$ are restored from $\mathrm{w}^{i+1}$ by using (3.8)

$$
\begin{aligned}
\mathbf{w}_{2}^{i} & =\left(D_{1}^{i}\right)^{-1}\left[\mathbf{r}_{1}^{i}-C_{1}^{j} \mathbf{w}_{1}^{i}-\mathbf{w}_{3}^{i}\right], \\
\mathbf{w}_{2 i}^{i}=\left(B^{i}\right)^{-1}\left[\mathbf{r}_{2 i-1}^{i}-\mathbf{w}_{2 i-3}^{i}-C^{i} \mathbf{w}_{2 i-1}^{i}-\mathbf{w}_{2 i+1}^{i}\right]-\mathbf{w}_{2 i-2}^{i}, & i=2,3, \cdots,\left(q_{j}-1\right) / 2 .
\end{aligned}
$$

To solve (3.7) by the factoring method, it follows from (3.1), (2.6), and (2.7) that we must store $3 q_{i+1}-2$ matrices, each of dimension $p^{2}$. In addition, we must store $8(j+1)$ matrices, of dimension $p^{2}$, of $(3.10)$ and (3.11). They are required to form $\mathbf{r}^{1}, \cdots, \mathbf{r}^{j+1}$ and to restore the eliminated subvectors. The elimination reduces the number of matrices for the factoring method at each step by $\left(q_{i}-1\right) / 2$. However, the addition of $8(j+1)$ new matrices makes the reduction uneconomical after very few steps. A further consideration is that the matrices may become ill-conditioned for $j$ sufficiently large.

4. Applications to Biharmonic Boundary-Value Problems. ${ }^{4}$ Problems $B_{1}$ and $B_{2}$ are defined by (1.2) on the unit square with

$$
\begin{aligned}
r(x, y) & =r_{1}(x, y) \\
& =8\left[3 y^{2}(1-y)^{2}+3 x^{2}(1-x)^{2}+\left(6 x^{2}-6 x+1\right)\left(6 y^{2}-6 y+1\right)\right], \\
r(x, y) & =r_{2}(x, y) \\
& =(2 \pi)^{4}[4 \cos 2 \pi x \cos 2 \pi y-\cos 2 \pi x-\cos 2 \pi y],
\end{aligned}
$$

respectively. The solutions are

$$
w_{1}=x^{2}(1-x)^{2} y^{2}(1-y)^{2}, \quad w_{2}=(1-\cos 2 \pi x)(1-\cos 2 \pi y) .
$$

Numerical solutions of Problems $B_{1}$ and $B_{2}$ were obtained by the factoring method with a mesh width of $\delta=1 / 26$ and the results were compared at the mesh points with (4.2). The pointwise error is defined as the difference between (4.2) and the numerical values, divided by the maximum value of (4.2). The maximum errors for Problems $B_{1}$ and $B_{2}$ occurred at the centèr $x=y=1 / 2$ and were $1.13 \%$ and $.98 \%$, respectively. Numerical solutions of Problems $\mathrm{B}_{1}$ and $\mathrm{B}_{2}$ were obtained also by using the 25-point difference approximation of the biharmonic operator. Little or no improvement was obtained in the accuracy.

To evaluate the speed of the method, we considered (1.2) on the unit square with

$$
r(x, y)=1 \text {. }
$$

The solution of this problem is unknown. Numerical solutions were obtained in [2] using the alternating direction method. We repeated this calculation with $\delta=1 / 26$ and the iteration parameters that are suggested in [2]. The convergence criterion was

- All the computations were performed on the CDC 6600 computer at the AEC Computing and Applied Mathematics Center of the Courant Institute of Mathematical Sciences. 


$$
\max _{i, j}\left|w_{i j}^{n}-w_{i j}^{n-1}\right|<\epsilon .
$$

Here, $w_{i j}$ is the mesh function corresponding to $w\left(x_{i}, y_{i}\right)$; the superscript on $w_{11}$ indicates the cycle number [2]; the maximum in (4.4) is taken over all mesh points; and $\epsilon$ is a prescribed small number. For $\epsilon=10^{-5}$, the difference in the results for the present and the alternating direction methods were less than $.03 \%$. For $\epsilon=10^{-9}$, better agreement was obtained. For $\epsilon=10^{-5}$ and $\epsilon=10^{-9}, 6.6$ and 11 seconds of computing time were required, respectively, to satisfy (4.4). By comparison, the unit inversion time for the factoring method is $\tau=.09$ seconds. Thus, if comparable accuracy is desired, the alternating direction method is 60 to 100 times slower than the factoring method. However, it is important to observe that finer mesh widths, e.g., $\delta=1 / 80$, can be employed with the alternating direction method. It was possible to obtain $\delta=1 / 32$ with the factoring method alone, and $\delta=1 / 40$ and $\delta=1 / 46$, using the block elimination procedure once and twice, respectively.

We have made the comparison with the alternating direction method since it seems to be the most efficient method previously presented in the literature for the solution of biharmonic boundary-value problems. Elementwise, Gauss elimination methods, using the sparseness of $M$ and its submatrices, might be competitive or superior to our method. However, applications of such methods to the system (1.1), for the size of $M$ that we consider, have not, to our knowledge, been reported in the literature.

5. Natural Frequencies and Modes of a Clamped, Square Plate. The natural frequencies and natural modes of vibration of a clamped elastic plate are obtained from the eigenvalues $\lambda$ and the eigenfunctions $w(x, y)$ of

$$
\begin{gathered}
\Delta^{2} w=\lambda w, \quad \text { for } x, y \text { in } R, \\
w=\mathbf{n} \cdot \nabla w=0, \text { for } x, y \text { on } B .
\end{gathered}
$$

We assume that the eigenfunctions are normalized so that

$$
\|w\|^{2} \equiv \iint w^{2} d x d y=1
$$

The integral in (5.2) and all subsequent integrals are over $R$. If $\lambda$ is an eigenvalue and $w$ is an eigenfunction of (5.1), then

$$
\lambda=\Lambda(w) \equiv \iint(\Delta w)^{2} d x d y .
$$

We denote the eigenvalues of (5.1), counting multiplicities, by $\lambda_{1}, \lambda_{2}, \cdots$, and the corresponding eigenfunctions by $w^{(1)}(x, y), w^{(2)}(x, y), \cdots$. We determine them numerically by an iterative procedure that is related to the power method [7]. Thus, we suppose that the first $n-1$ eigenvalues and eigenfunctions are known. Starting from an initial estimate $w_{0}^{(n)}$, we define a sequence of iterates $\lambda_{n, 1}, \lambda_{n, 2}, \cdots, \lambda_{n, k}, \cdots$ and $w_{1}^{(n)}, w_{2}^{(n)}, \cdots, w_{k}^{(n)}, \cdots$ by the recursions

$$
\Delta^{2} \bar{w}_{k+1}^{(n)}=\lambda_{n, k} w_{k}^{(n)}, \quad \bar{w}_{k+1}^{(n)}=\mathrm{n} \cdot \nabla \bar{w}_{k+1}^{(n)}=0 \quad \text { on } B,
$$




$$
\begin{aligned}
& \tilde{w}_{k+1}^{(n)}=\bar{w}_{k+1}^{(n)}-\sum_{i=1}^{n-1} a_{i}^{k+1} w^{(i)}, \\
& w_{k+1}^{(n)}=\tilde{w}_{k+1}^{(n)} /\left\|\tilde{w}_{k+1}^{(n)}\right\|, \quad k=0,1, \cdots,
\end{aligned}
$$

where $\lambda_{n, k}$ and $a_{i}^{k}$ are defined by

$$
\lambda_{n, k}=\Lambda\left(w_{k}^{(n)}\right), \quad a_{i}^{k+1}=\iint \bar{w}_{k+1}^{(n)} w^{(i)} d x d y, \quad j=1,2, \cdots, n-1 .
$$

Thus, $w_{k+1}^{(n)}$ is the normalized projection of the provisional iterate $\bar{w}_{k+1}^{(n)}$ into the orthogonal complement of the subspace spanned by $w^{(1)}, w^{(2)}, \cdots, w^{(n-1)}$. We employed the following convergence criterion for the iterations

$$
\left|\lambda_{n, k+1}-\lambda_{n, k}\right|<10^{-4} \text {. }
$$

Each iterate was numerically determined by solving (5.4a) by the factoring and block elimination methods. The integrals in (5.4) were evaluated by Simpson's rule. We applied the numerical method (5.4) to the unit square plate. Numerical approximations for each eigenvalue and eigenfunction were obtained for the three mesh widths

$$
\delta_{1}=1 / 32, \quad \delta_{2}=1 / 26, \quad \delta_{3}=1 / 20 .
$$

Richardson's mesh extrapolation procedure [7] was used to obtain an improved approximation of the eigenvalue. That is, if $\lambda_{n}(\delta)$ is the converged numerical value with mesh width $\delta$ for the $n$th eigenvalue, we assume that

$$
\lambda_{n}(\delta)=\lambda_{n}+a \delta^{2}+b \delta^{4}
$$

for some constants $a$ and $b$. Here, $\lambda_{n}$ is the Richardson approximation of the eigenvalue. We determine $\lambda_{n}$ by substituting the three values of $\lambda_{n}(\delta)$ and $\delta$ into (5.7). For $n<13$, (5.4a) was solved by the factoring method alone. For $n \geqq 13$, the block elimination procedure was required because of the additional storage needed for the projection calculations $(5.4 \mathrm{~b})-(5.4 \mathrm{~d})$.

The number of iterations that are required to satisfy (5.5) depends on the values of $\delta$ and $n$, and on the initial guess $w_{0}^{(n)}$. The number of iterations usually increases with $n$. From 7 to 31 iterations were required to satisfy (5.5). For $\delta=1 / 32$, the unit iteration time for the first eigenvalue was approximately .1 seconds. The unit iteration time increases with $n$ because of the additional projection calculations (5.4b) and (5.4d). The computation of 20 eigenvalues required a total of 381 iterations which used approximately 4 minutes of computing time. According to the estimates described in Section 4, the same results would require approximately 45 minutes if the alternating direction method were employed.

To test the accuracy of the numerical method (5.4)-(5.7), we applied it to the solution of the biharmonic eigenvalue problem consisting of (5.1a) on the unit square and the simply supported boundary conditions, $w=w_{n n}=0$. The eigenvalues and eigenfunctions of the simply supported plate are known explicitly. They are

$$
\lambda=\mu_{m n} \equiv\left(m^{2}+n^{2}\right)^{2} \pi^{4}, \quad w=2 \sin m \pi x \sin n \pi y .
$$

A comparison of the exact (5.8) and the numerically determined eigenvalues is presented in Table I. Similar accuracy need not occur for the clamped plate. We 


\section{TABLB I}

A Comparison of the Exact $\left(\mu_{n}^{1 / 2}\right)$ and the Numerically Determined Frequencies $\left(\hat{\mu}_{n}^{1 / 2}\right)$ of the Square, Simply Supported Plate

\begin{tabular}{clll}
\hline$n$ & \multicolumn{1}{c}{$\hat{\mu}_{n}^{1 / 2}$} & \multicolumn{1}{c}{$\mu_{n}^{1 / 2}$} & $\%$ Diff. \\
\hline 1 & 19.7392081 & 19.7392088 & .0000035 \\
2,3 & 49.348020 & 49.348022 & .000004 \\
4 & 78.956830 & 78.956835 & .0000063 \\
5,6 & 98.69598 & 98.69604 & .000061 \\
\hline
\end{tabular}

observe that the accuracy decreases as $n$ increases, i.e., as the number of waves in the eigenfunction increases.

The results for the clamped plate (5.1) are summarized in Table II. They are compared with the results of the Rayleigh-Ritz calculation in [4], [5] and the asymp-

\section{TABLE II}

Frequencies $\left(\lambda^{1 / 2}\right)$ of the Clamped, Square Plate Obtained by the Numerical Method $\left(\hat{\lambda}_{n}^{1 / 2}\right)$, the Ritz Method $(R)$, and an Asymptotic Method [Bolotin].

The exact frequencies $\left(\mu_{n}^{1 / 2}\right)$ of the simply supported plate are also given.

\begin{tabular}{clccccc}
\hline$n$ & \multicolumn{1}{c}{$\mu_{n}^{1 / 2}$} & \multicolumn{1}{c}{$\lambda_{n}^{1 / 2}$} & $R[4]$ & $\%$ Diff. & Bolotin [8] & $\%$ Diff. \\
\hline 1 & 19.7392 & 35.9844 & 35.99 & .016 & 35.096 & -2.47 \\
$2-3^{*}$ & $49.3480^{*}$ & 73.3894 & 73.41 & .028 & 72.897 & -.67 \\
4 & 78.9568 & 108.1986 & 108.27 & .066 & 107.47 & -.67 \\
5 & $98.6960^{*}$ & 131.5626 & 131.64 & .059 & - & - \\
6 & $98.6960^{*}$ & 132.1859 & 132.25 & .048 & 131.63 & -.42 \\
$7-8 *$ & $128.3049 *$ & 164.9468 & 165.15 & .123 & 164.39 & -.34 \\
$9-10^{*}$ & $167.7833^{*}$ & 210.4574 & & & 210.35 & -.051 \\
11 & 177.6529 & 219.8986 & & & 219.32 & -.26 \\
12 & $197.3921^{*}$ & 242.0102 & & & - & - \\
13 & $197.3921^{*}$ & 243.0087 & $243.10[5]$ & .038 & 242.20 & -.33 \\
$14-15^{*}$ & $246.7401^{*}$ & 296.039 & & & 295.69 & -.12 \\
16 & $256.6097^{*}$ & 308.707 & & & - & - \\
17 & $256.6097^{*}$ & 308.974 & & & 308.929 & -.015 \\
$18-19 *$ & $286.2185^{*}$ & 340.249 & & & 340.244 & -.0015 \\
20 & 315.8273 & 370.761 & & & 370.66 & -.027 \\
21 & $335.5665^{*}$ & 392.130 & & & 392.80 & -.13 \\
22 & $335.5665^{*}$ & 393.326 & & & & \\
\hline
\end{tabular}

totic approximations of Bolotin [8]. The eigenvalues of the simply supported plate are also shown in Table II. Of course, there may be other eigenvalues between the ones shown in Table II that we failed to determine with our numerical methods. Multiple eigenvalues are denoted by an asterisk. All multiple eigenvalues that were found had multiplicity two. Multiple eigenvalues were not discussed in [4], [5], [8]. 
5.1. Nodal Properties of the Eigenfunctions. Perspective sketches of the numerically determined eigenfunctions for the clamped, square plate are shown in Fig. 1. Their nodal lines are shown in Fig. 2. We shall refer to the eigenfunction

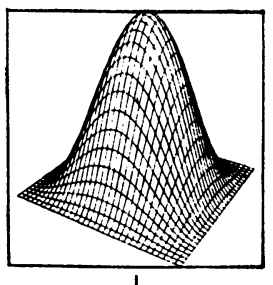

I

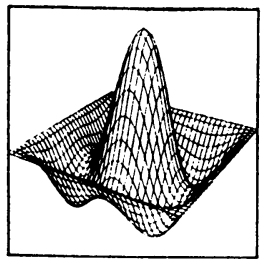

6

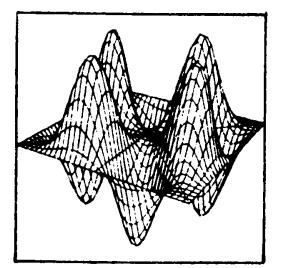

12

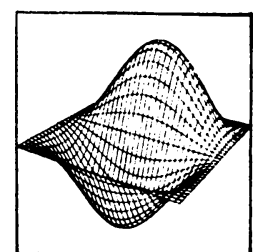

$2-3$

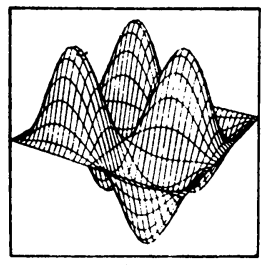

$7-8$

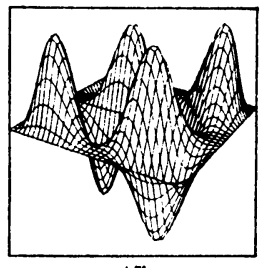

13

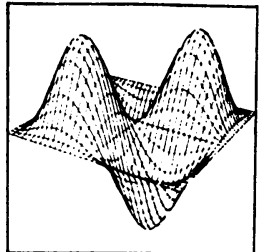

4

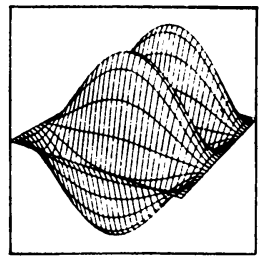

$9-10$

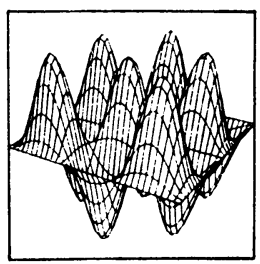

14-15

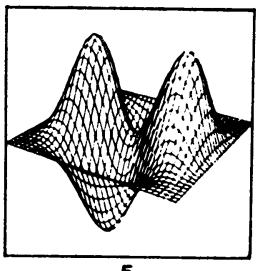

5

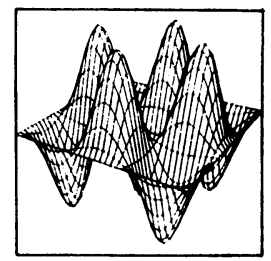

II

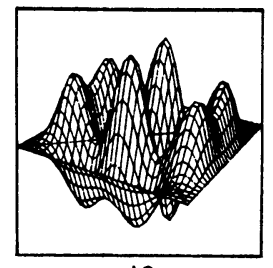

16

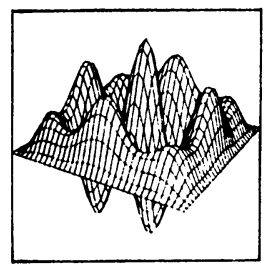

17

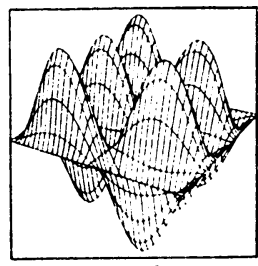

$18-19$

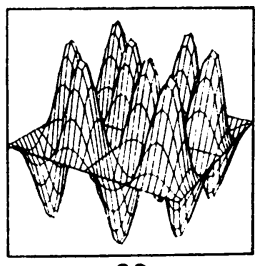

20
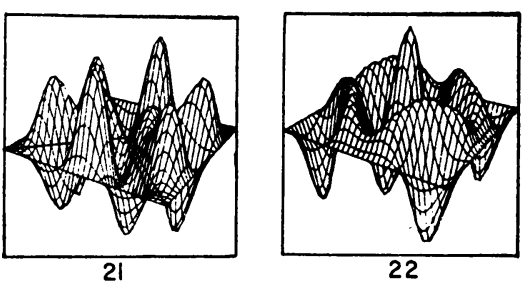

FigURE 1. Computer made sketches of the numerically determined eigenfunctions. The mode number is indicated under each sketch. For each double eigenvalue, only one eigenfunction is shown. The other eigenfunction is obtained by rotating the figure through ninety degrees about a vertical axis through the center.

corresponding to the lowest eigenvalue as the principal eigenfunction. It is important to observe that the numerically determined principal eigenfunctions for the meshes $\delta=1 / 32$ and $\delta=1 / 26$ have nodal curves near the corners of the plate. ${ }^{5}$ The existence of nodal curves for the principal eigenfunction was not reported in [4], [5], [8]. Since these nodal curves are close to the corners, we further studied their occurrence by three special computations. First, we solved (5.1) by the iterative method (5.4)-(5.7)

${ }^{5}$ The principal eigenfunction for the simply supported plate is free of nodes; see (5.8). 


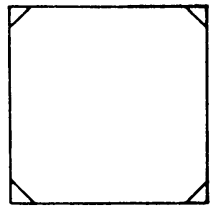

I

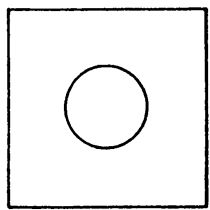

6

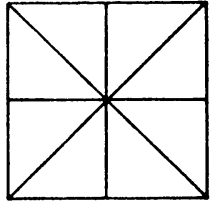

12

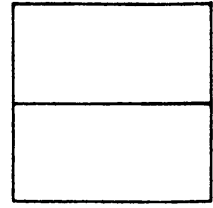

$2-3$

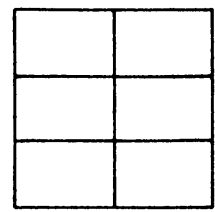

$7-8$

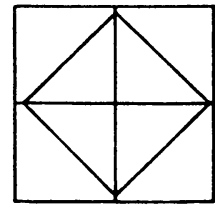

13

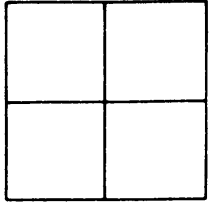

4

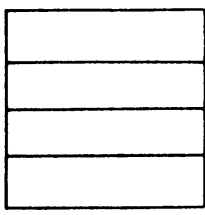

$9-10$

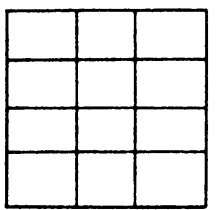

14-15

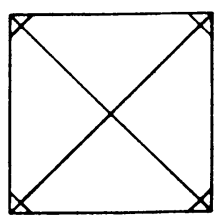

5

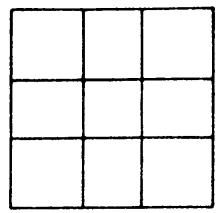

II

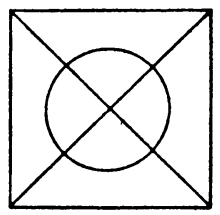

16

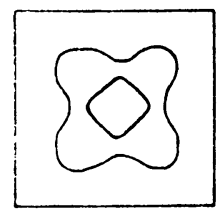

17

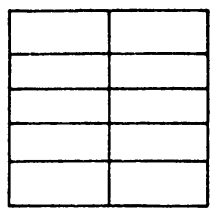

$18-19$

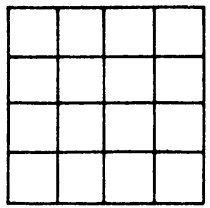

20

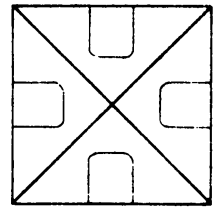

21

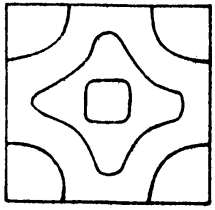

22

FIGURE 2

The nodal lines for the eigenfunctions sketched in Fig. 1.

using a $\delta=1 / 80$ mesh and the alternating direction method. We found that the nodal curves extended to approximately $1 / 20$ of the length of a side. For this mesh, there were a total of 10 mesh points between each nodal curve and the corner. The amplitudes of the solutions in these corner regions were small and opposite in sign to the solution in the center of the plate.

In the second computation, we used the numerical method to determine the lowest eigenvalue $\lambda$ and principal eigenfunction of

$$
\Delta^{2} w-T \Delta w=\lambda w, \quad w=\mathrm{n} \cdot \nabla w=0 \quad \text { on } B .
$$

The eigenvalue problem describes the flexural vibrations of stretched, clamped, square elastic plates [9]. $T$ is the prescribed uniform stretch on the boundary of the plate. When $T=0,(5.9)$ is reduced to (5.1). If $T<0$, the plate is uniformly compressed. We determined the principal eigenfunction of (5.9) numerically for a de- 
creasing sequence of $T$ values starting at $T=5 \pi^{2}$. For $\delta=1 / 32$, it was free of nodes for $T=5 \pi^{2}$. For $T<44$, nodal curves occurred in the corners. The nodal curves moved very slowly into the interior of the plate and the amplitude near the corners increased as $T$ decreased. For $T=-50$, the nodal curves extended to approximately $3 / 32$ the length of the edge. ${ }^{6}$

Finally, we considered the intermediate eigenvalue problem,

$$
\Delta^{2} w=\lambda w, \quad w=(1-\epsilon) w_{n n}+\epsilon n \cdot \nabla w=0 \text { on } B,
$$

for the square plate. The boundary conditions in (5.10) correspond to an edge that is elastically restrained against rotation. When $\epsilon=0$ or $\epsilon=1,(5.10)$ is the eigenvalue problem for the simply supported or the clamped plate, respectively. The parameter $\epsilon$ is related to the rotational spring constant of the edge. We determined the principal eigenfunction of (5.10) numerically for a sequence of values of $\epsilon$. The principal eigenfunction was free of nodes for $\epsilon<.996$ and corner nodal curves appeared for $\epsilon \geqq .996$. Thus, the occurrence of nodal curves depends strongly on the rotational stiffness of the boundary.

The computations demonstrate the existence of nodal curves for the principal eigenfunction of (5.1) for the square plate. Since the results are numerical and the amplitudes are small near the corners, a rigorous proof of the existence of the nodal curves is still required. ${ }^{7}$ We recall that Weyl and Courant [10] have shown for a class of second-order elliptic eigenvalue problems (including the vibrating membrane) that the principal eigenfunction is always free of nodes.

Duffin and Shaffer [11] announced that for the vibrating annular plate with clamped inner radius $r_{0}$ and clamped outer radius 1 , the principal eigenfunction has a diametral nodal line if $r_{0}$ is sufficiently small. Specifically, they found that $r_{0}$ must be $<1 / 715$. This seems to be the only previous example of a domain for which the principal eigenfunction of the mathematical problem (5.1) has nodes.

Rayleigh [12] conjectured and Faber [13] proved that, of all vibrating membranes of given area, the circular membrane possesses the smallest principal eigenvalue. Szegö [14] proposed that a similar result holds for (5.1), i.e., of all clamped vibrating plates of given area, the circular plate has the smallest principal eigenvalue. To prove this theorem, Szegö assumed that the principal eigenfunction of (5.1) has no internal nodes. Our results suggest that this assumption is invalid and that the proof is incomplete, although the theorem may be correct.

5.2. Properties of the Spectrum. We observe that the eigenfunctions shown in Figs. 1 and 2 may be classed in three groups according to the properties of their nodal lines:

(1) Eigenfunctions 1, 4, 11 and 20 have an equal number of nodal lines parallel to the $x$ and to the $y$ axis. They correspond to simple eigenvalues.

(2) Eigenfunctions 2-3, 7-8, 9-10, 14-15 and 18-19 have nodal lines parallel to the $x$ and $y$ axis. However, the number of nodal lines, $N_{x}$ and $N_{y}$, in the two directions is different. In addition, $\left(N_{x}+N_{y}\right)$ is odd. They correspond to double eigenvalues.

B $T \approx-52.34$ is the lowest eigenvalue of the eigenvalue problem (5.9) with $\lambda=0$.

7 A related problem is to characterize the domains for which the principal eigenfunctions of (5.1) have nodes or are free from nodes. Additional numerical results are discussed in the note added in proof at the end of the paper. 
(3) The remaining eigenfunctions in Figs. 1 and 2 can be grouped in the pairs, 5-6, 12-13, 16-17, and 21-22. The eigenvalues corresponding to each pair are simple but close in value (see Table II). We refer to them as weakly simple pairs. The member of each pair with the lower eigenvalue has two diagonal nodal lines. For even values of $N_{x}+N_{y}$, eigenfunctions only with nodal lines parallel to the $x$ and $y$ axis were not determined. Bolotin's method fails to approximate the eigenvalues corresponding to the eigenfunctions with two diagonal nodal lines.

We observe, by comparing the eigenvalues of the clamped and simply supported plates in Table II, that to every simple eigenvalue of the simply supported plate, there corresponds a simple eigenvalue of the clamped plate. To every double eigenvalue of the clamped plate, there corresponds a double eigenvalue of the simply supported plate. However, every double eigenvalue of the simply supported plate need not correspond to a double eigenvalue of the clamped plate. In fact, we see from Table II that there are double eigenvalues of the simply supported plate, e.g., $n=5$ and 6 , which apparently split to form weakly simple pairs of eigenvalues of the clamped plate.

To further study the splitting, we numerically determined the variation with $\epsilon$ of the eigenvalues of (5.10) which coalesce to the double eigenvalue $n=5,6$ for $\epsilon=0$ and split to the weakly simple pair $n=5$ and $n=6$ for $\epsilon=1$. The results are summarized in Table III. We observe that the double eigenvalue apparently

\section{TABLE III}

The Splitting of a Double Eigenvalue of the Simply Supported Plate into a Weakly Simple Pair for the Clamped Plate

\begin{tabular}{ccc}
\hline & $n=5$ & $n=6$ \\
\hline 0 & 9740.896 & 9740.896 \\
.1 & 9741.134 & 9741.113 \\
.2 & 9742.088 & 9741.990 \\
.3 & 9744.349 & 9744.075 \\
.5 & 9758.761 & 9757.518 \\
.9 & 10511.620 & 10507.691 \\
.99 & 15113.570 & 15244.616 \\
1.0 & 17308.092 & 17472.486 \\
\hline
\end{tabular}

splits immediately for $\epsilon>0$. For $\epsilon \approx .91$, the two eigenvalue branches intersect again.

6. Natural Frequencies of Clamped Cylindrical Panels. We consider a thin circular cylindrical panel of radius of curvature $a$, thickness $h$, axial length $L$ and circumferential length $a \theta_{0}$. The natural frequencies and modes of the panel are obtained from the eigenvalues $\lambda$ and eigenvectors $[w(x, y), f(x, y)]$ of

$$
\Delta^{2} w=K f_{x x}+\lambda w, \quad \Delta^{2} f=-w_{x x}, \text { for } x, y \text { in } R,
$$


(6.1b)

$$
w=\mathrm{n} \cdot \nabla w=f=\mathrm{n} \cdot \nabla f=0, \quad \text { for } x, y \text { on } B .
$$

The parameter $K$ is defined by

$$
K \equiv \frac{12\left(1-\nu^{2}\right)\left(a^{2} \theta_{0}^{2} / h\right)^{2}}{a^{2}}
$$

The region $R$ is the rectangle: $0 \leqq y \leqq 1,0 \leqq x \leqq l \equiv L /\left(a \theta_{0}\right)$, where $x$ and $y$ are dimensionless axial and circumferential coordinates. The conditions (6.1b) imply that the boundary is clamped and stress-free with respect to the midsurface stresses. If $K=0$, e.g., $a \rightarrow \infty$ with $\left(a \theta_{0}\right)^{2} / h$ fixed, the differential equations in (6.1a) uncouple. The first equation in (6.1) is then equal to (5.1a).

We assume that the eigenfunctions of (6.1) are normalized so that

$$
\|w\|^{2}=\iint w^{2} d x d y=1
$$

The eigenvalues and eigenfunctions are related by

$$
\lambda=\Lambda_{K}(w, f) \equiv \iint\left[(\Delta w)^{2}+K(\Delta f)^{2}\right] d x d y .
$$

We observe that $\Lambda_{0}=\Lambda$, which is defined in (5.3).

Approximations of the eigenvalues and eigenvectors of (6.1) were obtained by iterative and numerical methods analogous to the ones used in the previous section for the flat plate. For simplicity, we shall only consider the lowest eigenvalue. Thus, we define a sequence of iterates $\lambda_{1,1}, \lambda_{1,2}, \cdots,\left(w_{1}, f_{1}\right),\left(w_{2}, f_{2}\right), \cdots$ by the recursions

$$
\begin{aligned}
\Delta^{2} f_{p} & =-w_{p, x x}, & \Delta^{2} \bar{w}_{p+1} & =K f_{p, x x}+\lambda_{1, p} w_{p}, \\
\lambda_{1, p} & =\Lambda_{K}\left(w_{p}, f_{p}\right), & w_{p+1} & =\bar{w}_{p+1} /\left\|\bar{w}_{p+1}\right\| .
\end{aligned}
$$

All the calculations were made for the square panel, $l=1$. The Richardson approximation of the eigenvalues, $\lambda_{1}$, are summarized in Table IV. Usually, eight

\section{TABLE IV}

The Lovest Eigenvalue for the Clamped, Square, Cylindrical Panel Obtained by the Numerical $\left(\hat{\lambda}_{1}\right)$ and Perturbation $(P)$ Methods [see (A.7)].

\begin{tabular}{rccc}
\hline$K$ & $\hat{\lambda}_{1}$ & $P$ & $\%$ Diff. \\
\hline 0 & 1294.88 & - & - \\
$10^{2}$ & 1306.50 & 1306.52 & .0015 \\
$10^{3}$ & 1410.20 & 1411.24 & .074 \\
$2 \times 10^{3}$ & 1523.54 & 1527.60 & .266 \\
\hline
\end{tabular}

iterates were required for convergence. The unit iteration time is slightly larger for this problem since it involves the solution of two biharmonic problems at each step.

In Table IV, we also list the approximations of $\lambda_{1}$ obtained by a perturbation expansion for small $K$, i.e., an expansion in the neighborhood of the flat plate. The perturbation method is described in Appendix A. The agreement between the perturbation and numerical methods is excellent even for "large" values of $K$. The 
results indicate that the lowest eigenfunction also has nodal curves near the corners of the panel.

Appendix A. The Perturbation Method for the Shallow Cylindrical Panel. We assume that for small $K$ the lowest eigenvalue and eigenvector of (6.1) are analytic in $K$, i.e.,

$$
\lambda_{1}(K)=\sum_{i=0}^{\infty} \lambda^{(i)} K^{i}, \quad\left(\begin{array}{l}
w \\
f
\end{array}\right)=\sum_{i=0}^{\infty}\left(\begin{array}{c}
w^{(i)} \\
f^{(i)}
\end{array}\right) K^{i} .
$$

The coefficients $\lambda^{(i)}, w^{(i)}$ and $f^{(i)}$ are determined by inserting (A.1) into (6.1a), (6.1b) and equating coefficients of the same powers of $K$. This gives, for $i=0,1, \cdots$,

$$
\begin{gathered}
\Delta^{2} w^{(i)}-\lambda^{(0)} w^{(i)}=F^{(i)} \equiv f_{x x}^{i-1}+\sum_{j=1}^{i} \lambda^{(i)} w^{(i-i)}, \\
w^{(i)}=\mathrm{n} \cdot \nabla w^{(i)}=0 \quad \text { on } B,
\end{gathered}
$$

and

$$
\begin{gathered}
\Delta^{2} f^{(i)}=-w_{x x}^{(i)}, \\
f^{(i)}=\mathrm{n} \cdot \nabla f^{(i)}=0 \text { on } B,
\end{gathered}
$$

where we define $w^{(-1)}=f^{(-1)} \equiv 0$. For $i=0,\left(\right.$ A.2) is equal to (5.1). Thus, $\lambda^{(0)}=$ $\lambda_{1}(0)$ and $w^{(0)}$ are the lowest eigenvalue and normalized eigenfunction of the clamped flat plate for which accurate numerical approximations are known. Since $\lambda^{(0)}$ is an eigenvalue of (4.1), each $F^{(i)}, i=1,2, \cdots$, must satisfy the solvability conditions

$$
\iint F^{(i)} w^{(0)} d x d y=0 .
$$

In evaluating $F^{(i)}$, we consider, from (A.3), $f^{i-1}(x)$ as a functional of $w^{(i-1)}$. Thus, $F^{(i)}$ depends only on $w^{(0)}, w^{(1)}, \cdots, w^{(i-1)}$. For $i=1$ in (A.4), we find, by using (A.3) and integration by parts, that $\lambda^{(1)}$ is given by

$$
\lambda^{(1)}=\iint\left[\Delta f^{(0)}\right]^{2} d x d y .
$$

Here, $f^{(0)}$ is the solution of (A.3) with $i=0$. It is determined numerically by the factoring method using the numerical eigenfunction $w^{(0)}$. Thus, from (A.1), we have the following estimate for $\lambda_{1}$ :

$$
\lambda_{1}(K) \approx \lambda^{(0)}+\lambda^{(1)} K .
$$

For the square plate, we obtain $\lambda^{(1)} \approx .1163611$, and

$$
\lambda(K) \approx 1294.88+(.1163611) K .
$$

Notes Added in Proof. 1. We have numerically solved the eigenvalue problem (5.1) for the clamped, rhombic plate with a $60^{\circ}$ corner angle using the methods described in Section 5. The numerical principal eigenfunction had nodal curves near the $60^{\circ}$ corners and no nodal curves near the $120^{\circ}$ corners. The nodal curves occurred further away from the corners for the rhombic plate than for the square plate. The 
results suggest that corner nodal curves disappear at obtuse angles and become more pronounced near acute angles. Furthermore, we observed that corner nodal curves occur in the first 9 modes for the rhombic plate.

2. In an attempt to prove our results concerning the existence of nodal curves for the principal eigenfunction of (5.1), Professor S. Osher has proved that the Green's function for (5.1) changes sign near the corner. This is a necessary condition for the existence of corner nodal curves for the principal eigenfunction.

Courant Institute of Mathematical Sciences

New York University

New York, New York 10012

Department of Mathematics

New York University

University Heights

New York, New York 10453

1. S. SCHECHTER, "Quasi-tridiagonal matrices and type-insensitive difference equations," Quart. Appl. Math., v. 18, 1960/61, pp. 285-295. MR 22 \#5133.

2. S. D. CONTE \& R. T. DAMES, "On an alternating direction method for solving the plate problem with mixed boundary conditions," J. Assoc. Comput. Mach., v. 7, 1960, pp. 264-273. MR 26 \#2028.

3. B. L. Buzbee, G. H. Golub \& C. W. Nielson, "On direct methods for solving Poisson's equations," SIAM J. Numer. Anal., v. 7, 1970, pp. 627-656.

4. D. YounG, "Vibration of rectangular plates by the Ritz method," J. Appl. Mech., v. 17,1950 , pp. $448-453$.

5. S. IGUCHI, "Die Biegungsschwingungen der Vierseitig Eingespannten Rechteckigen Platten," Ing. Arch., v. 8, 1937, pp. 11-25.

6. L. BAUER \& E. L. REISS, "On the flexural vibrations of clamped orthotropic plates." (Submitted.)

7. E. Isaacson \& H. B. Keller, Analysis of Numerical Methods, Wiley, New York, 1966. MR 34 \#924.

8. V. V. Bolorin, "An asymptotic method for the study of the problem of eigenvalues for rectangular regions," in Problems of Continuum Mechanics (Muskhelishvili Anniversary Volume), SIAM, Philadelphia, Pa., 1961, pp. 56-68. MR 25 \#3631.

9. A. WEINSTEIN \& W. Z. CHIEN, "On the vibrations of a clamped plate under tension," Quart. Appl. Math., v. 1, 1943, pp. 61-68. MR 5, 98.

10. R. Courant \& D. Hilbert, Methods of Mathematical Physics. Vol. I, Interscience, New York, 1953. MR 16, 426.

11. R. J. DUfFIN \& D. H. SHAFFER, "On the modes of vibration of a ring shaped plate," Bull. Amer. Math. Soc., v. 58, 1952 , p. 652.

12. J. W. S. RaYleigh, The Theory of Sound, 2nd ed., Dover, New York, 1945. MR. 7, 500 .

13. G. FABER, "Beweis, dass unter allen homogenen Membranen von gleicher Fläche und gleicher Spannung die kreisförmige den tiefsten Grundton gibt," S.-B. Bayer. Akad. Wiss., 1923, pp. 169-172.

14. G. Szegö, “On membranes and plates," Proc. Nat. Acad. Sci. U.S.A., v. 36, 1950, pp. 210-216. MR 11, 757 . 